\title{
A Multi-objective Optimization Behavior Fusion Avoidance Method for Snake-like Rescue Robot
}

\author{
Li Hongyan ${ }^{1, *}$, Hou Yuanbin ${ }^{1}$ \\ ${ }^{1}$ Xi'an University of Science and Technology, College of Electrical and Control Engineering, 710054 Shaanxi Xi'an, China
}

\begin{abstract}
The obstacle avoidance path planning of rescue robot has been the key problem of autonomous navigation for rescue robot. Most of the existing path planning algorithms is aimed at the optimization of a particular target, often resulting in deadlock or oscillation in order to obtain the optimal solution or the optimal path. Therefore, using the multi-objective optimization theory, this paper presents a multi-objective optimization dynamic obstacle avoidance algorithm based on interval weights, the fusion process of obstacle avoidance process is divided into three seed action, by giving the behavior function of different weights, the output rate of real-time dynamic change of different action. This is not to obtain the optimal solution or the optimal path at the current moment, but to obtain only the most efficient solution or the most satisfactory path at the current moment. Experiments show that the algorithm can effectively improve the flexibility and security of the obstacle avoidance process under the premise of ensuring the real-time and robustness of the search and rescue robot obstacle avoidance process.
\end{abstract}

\section{Introduction}

Snake-like robot is one of the branches of bionic robot in recent years. Unlike robots that traditionally use wheels, legs, or tracks, the snake-like robot can imitate the movements of snake, and realize the no limb movement through the twisting and stretching of the body. It has the advantages of good stability, small cross sectional area and high flexibility.

Autonomous obstacle avoidance is an important part of autonomous navigation for snake-like rescue robot. Nowadays, scholars have put forward many different obstacle avoidance theories according to different navigation environment. The typical methods include Curvature rate method ${ }^{[1]}$,Vector field histogram method $^{[2-3]}$, Dynamic window method ${ }^{[4-5]}$, Neighborhood histogram $\operatorname{method}^{[6]}$, Limit cycle method ${ }^{[7]}$, these methods can improve the robustness of obstacle avoidance in different degrees, but they all need time to calculate the optimal path or the optimal solution, it will sacrifice some time. Even deadlock or shock phenomenon, affect the real-time robot obstacle avoidance.

Therefore, this paper proposes a real-time obstacle avoidance algorithm based on multi-objective optimization for snake-like rescue robot. This algorithm does not need to calculate the current optimization path, but only the most efficient solution or the most satisfactory path is needed. Under the premise of safe obstacle avoidance, the real-time and robustness of the obstacle avoidance process are effectively improved.

\section{Obstacle avoidance process behavior function}

The obstacle avoidance process of snake-like rescue robot can be divided into three sub behaviors: fast forward, keep target direction and real-time obstacle avoidance. In the process of obstacle avoidance navigation, the robot determines the most appropriate behavior function at the current moment according to the weights of each sub behavior and the priority parameters, so as to ensure the security and stability of the obstacle avoidance process.

\subsection{Move forward quickly}

The purpose of this behavior function is to make the snake-like rescue robot move to the target as fast as possible. Its objective function is a linear function about the translational speed, moving forward rapidly, and the sub function can be expressed as $b_{m f}(v, \omega)=a\left(v / v_{\max }\right)+b$.In the formula, $\mathrm{V}$ is the velocity, and $\omega$ is angular velocity. When the empirical constant $a=1, b=0$, the range of the target function varies between $[0,1]$, and is directly proportional to the $\mathrm{V}$ value, irrespective of the angular velocity $\omega$.

\subsection{Keep the target direction}

Keeping the target direction behavior function requires the robot to keep the same direction as the target in the 
process of obstacle avoidance so as to ensure that the robot can accomplish the task in the shortest distance. The higher the value of the behavior function, the more likely the robot will be in the same direction with the target at the current moment. Conversely, the less likely the same direction is. Thus, the behavior function that maintains the target direction selects a $\Pi$ function as the behavior map, that is, $\Pi(x)=1 /\left\{1+[(x-\gamma) / \beta]^{2}\right\}$. In the formula, the angle between the forward direction and the target direction of the robot is determined by $\gamma$, which determines the extremum of the function; $\beta$ is the empirical constant, which determines the size of the function graph window. In the function curve shown in figure $1, \gamma=0^{\circ}, \beta=45^{\circ}$.

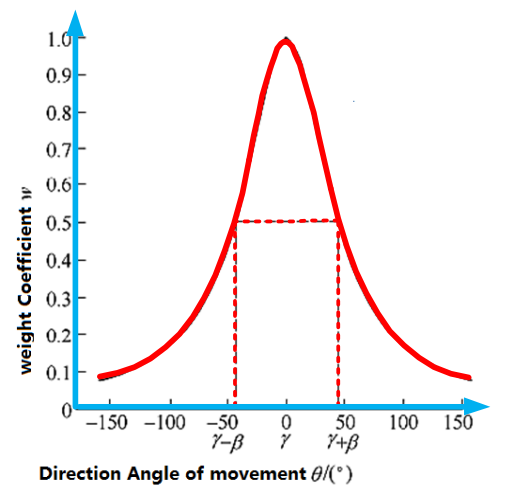

Fig. 1. Plot of the $\Pi$ function

According to the target position, the snake-like rescue robot determines the moving direction of each time toward the target, and its behavior function can be expressed

$$
\text { as } b_{m h}(v, \omega)=\Pi(\theta(v, \omega))=1 /\left\{1+\left[\left(\theta(v, \omega)-\theta_{\text {target }}\right) / \beta\right]^{2}\right\} \text {. }
$$

In the formula, $\theta=\theta_{0}+\omega T$ indicates that the robot is expected to move in the direction of $(v, \omega)$ control, $\theta_{0}$ is the initial direction of motion, $\mathrm{T}$ is the control instruction cycle, and $\theta_{t \text { arget }}$ is the direction of the robot when it reaches the target point. If the higher the objective function value is, the more likely the robot will be in the same direction as the target points at the current moment; otherwise, the smaller the likelihood is.

\subsection{Real-time obstacle avoidance}

The purpose of obstacle avoidance for snake-like rescue robot is to make the robot bypass the obstacle safely and steadily. Figure2 illustrates the obstacle avoidance process of a robot passing through any set of static obstacles.

A snake-like rescue robot can be viewed as a cylinder in which $\mathrm{R}$ is the radius of the robot cylinder; $A\left(x_{0}, y_{0}\right)$ is the center of the robot arc motion; $\varphi$ is the angle between the A point to the coordinate origin line and the A point to the barrier $P_{i}(x, y) ; d_{i}$ is the barrier spacing, and the $\mathrm{D}$ is the actual distance from the robot to the obstacle. The obstacle avoidance behavior function can determine a set of control parameters $(v, \omega)$ with the shortest safe path for a robot according to the minimum distance of a set of distance obstacles. The obstacle avoidance behavior function can be defined as

$$
b_{a o}(v, \omega)=\min \left\{D\left(v, \omega, d_{i}\right) \mid i \in N\right\}
$$

Considering the size of the robot and the position of the obstacle, the obstacle avoidance function can be expressed by piecewise function:

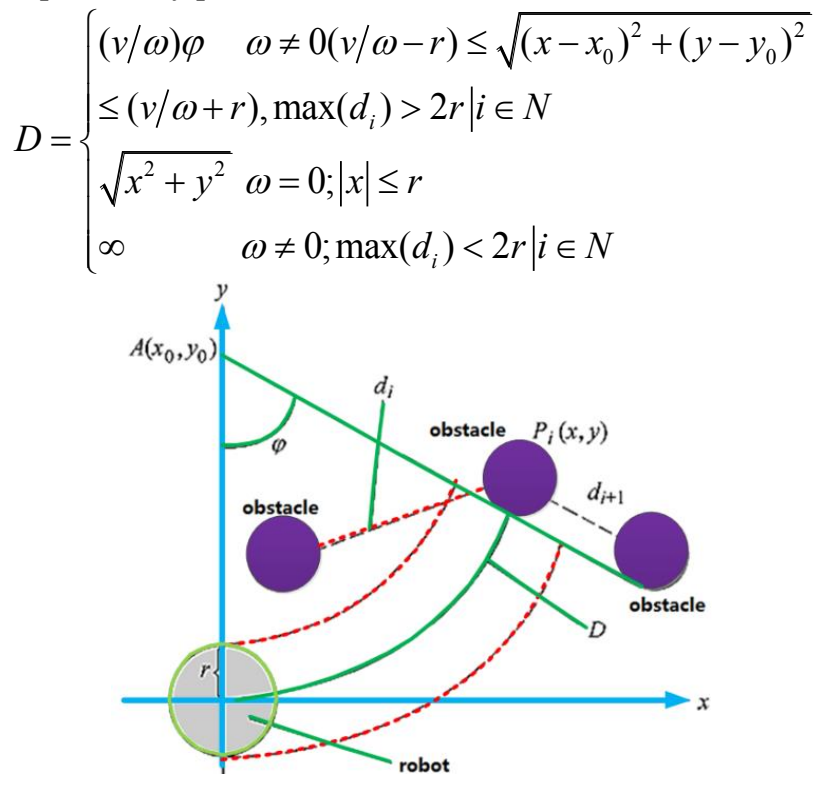

Fig. 2. Plot of obstacle avoidance process

The obstacle avoidance trajectory of snake-like rescue robot is related to $\mathrm{V}, \omega$, obstacle position $\mathrm{P}$, and location and size of robot itself, Therefore, the segmentation function of robot obstacle avoidance function is discussed.

The first segment value in the segmentation function represents the arc path of the robot to make the arc motion. At this point, the robot moves in an arc to the front of the obstacle, sensing the distance information of the obstacle in front of the airborne infrared sensor, and adopting the appropriate obstacle avoidance strategy;

The second segment indicates that the robot makes a uniform rectilinear motion trajectory. At this point, the obstacle is very close to the robot, and the robot's actual moving distance is the distance between the obstacle and the coordinate origin;

The third segment indicates that the robot airborne sensor detects very small distances between the obstacles and the robot body cannot pass through the barrier.

\section{Multi-objective optimization problem implementation}

The simultaneous optimization problem with multiple objectives (or indexes) is called a multi-objective optimization problem or a vector extremum problem. From the point of view of mathematical theory, the multi-objective optimization problem can be defined as follows

$\max \left[o_{1}(x), o_{2}(x), \cdots o_{n}(x)\right] \quad x=\left(x_{1}, x_{2}, \cdots x_{N}\right) \in R^{N}$ 
In the formula, $\mathrm{x}$ is a set of feasible action sets, and $o_{n}(x)$ is the action set credibility. According to the multi-objective optimization method, the navigation process of an autonomous mobile robot specifying the starting and ending points can be decomposed into three sub actions, namely, obstacle avoidance, keeping the target direction and moving rapidly toward the target. As shown in figure 3.

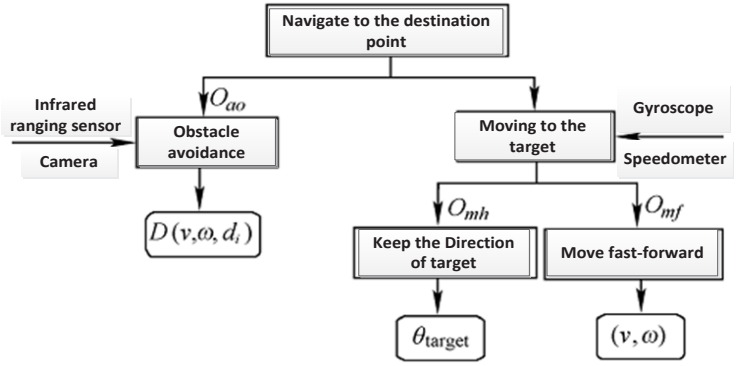

Fig. 3. specifies the navigation process behavior decomposition of the target

According to the decision theory, the weights, priorities and other factors are taken into account. A weighted objective programming method with dynamic interval weight is proposed to obtain the satisfactory solution of the system at present:

$$
\begin{aligned}
& \left(v^{*}, \omega^{*}\right)=\underset{(v, \omega) \in R^{N}}{\arg \max }\left[w_{m f} b_{m f}(v, \omega)+w_{m h} b_{m h}(v, \omega)+w_{a o} b_{a o}(v, \omega)\right] \\
& w_{m f}+w_{m h}+w_{a o}=1, w_{m f} \in[o, 0.1], w_{m h} \in[o, 0.4], w_{a o} \in[o, 1] .
\end{aligned}
$$

In the formula, $w_{m f}, w_{m h}, w_{a o}$ respectively represent fast

forward, keep the target direction and the obstacle avoidance behavior of three sub function weights.

The weighted values of the sub behaviors of the algorithm can be adjusted dynamically within a given interval, so as to ensure that the mobile robot can obtain the most efficient solution in real time.

\section{Reliability analysis of multi output obstacle avoidance system}

Definition1(multiple behavior output system).If a control system with $\mathrm{n}$ behavioral parameters has at least $\mathrm{k}$ successful output, and then the system is called $k-o u t-o f-n$ system $^{[8]}$. If the outputs of each subsystem in the system are independent of each other, the reliability function of the system can be expressed as:

$$
\begin{aligned}
& R\left(p_{1}, \cdots, p_{n}\right)=P\left\{X_{i}=1 \mid i=k, \cdots, n\right\}=\sum_{l=k}^{n} \sum_{x \in \Omega(l, \mathrm{n})}\{\psi(x) \mid k=1,2, \cdots, n\} \\
& =\sum_{l=k}^{n} \sum_{x \in \Omega(l, \mathrm{n})}\left\{\prod_{i=1}^{n} \Delta_{i}(x) \mid k=1,2, \cdots, n\right\}
\end{aligned}
$$

In the formula, $\psi(x)$ represents the probability that $x \in \Omega(k, \mathrm{n})$ is satisfied. $\Omega(k, \mathrm{n})$ represents all subsets of $\mathrm{k}$ behaviors that are successfully output in $\mathrm{n}$ behavior. If the system succeeds in output, then the $\mathrm{k}$ behavior value is 1 , and the $n-k$ behavior value is 0 .
For example, $\Omega(2,3)=\{(1,1,0),(1,0,1),(0,1,1)\}$, the function $\Delta:\{0,1\}^{n} \rightarrow[0,1]$ is defined as

$$
\Delta_{i}(x)= \begin{cases}P_{i} & x(i)=1 \\ 1-P_{i} & \text { otherwise }\end{cases}
$$

Aiming at the snake-like rescue robot obstacle avoidance system (2-out-3) with 3 sub outputs, the independence of obstacle avoidance behavior is analyzed, if the behavior of reliability for $P_{1}, P_{2}, P_{3}$, the formula (1) can be used to calculate the system reliability ${ }^{[9-10]}$.

$$
\begin{aligned}
& R\left(p_{1}, p_{2}, p_{3}\right)=\sum_{l=2}^{3} \sum_{b \in \Omega(l, 3)} \prod_{i=1}^{3} \Delta_{i}(b)=\sum_{b \in \Omega(2,3)} \prod_{i=1}^{3} \Delta_{i}(b)+\sum_{b \in \Omega(3,3)} \prod_{i=1}^{3} \Delta_{i}(b) \\
& =\sum_{b \in\{(1,1,0),(1,0,1),(0,1,1)\}} \prod_{i=1}^{3} \Delta_{i}(b)+\sum_{b \in\{(1,1,1)\}} \prod_{i=1}^{3} \Delta_{i}(b) \\
& =\prod_{i=1}^{3} \Delta_{i}(1,1,0)+\prod_{i=1}^{3} \Delta_{i}(1,0,1)+\prod_{i=1}^{3} \Delta_{i}(0,1,1)+\prod_{i=1}^{3} \Delta_{i}(1,1,1) \\
& =p_{1} p_{2}\left(1-p_{3}\right)+p_{1}\left(1-p_{2}\right) p_{3}+\left(1-p_{1}\right) p_{2} p_{3}+p_{1} p_{2} p_{3}
\end{aligned}
$$

If $X$ represents the successful output of child behavior in $n$ behavior, the reliability of the multi output system can be expressed as

$$
R\left(p_{1}, \cdots, p_{n}\right)=\sum_{l=k}^{n} P\{X=l\}
$$

Which contains the parameter $(n, p)$ binomial random variable function $\mathrm{p}$ the probability form can be expressed as $p(i)=P\{X=i\}=\left(\begin{array}{l}n \\ i\end{array}\right) p^{i}(1-p)^{n-i}, \quad i=0,1, \cdots, n$. When $p=p_{1}=p_{2}=\cdots=p_{n}$, a binomial random variable with parameters $(n, p)$ can describe the reliability of a multi output system.

Theorem 1: for a given behavior set, the reliability of $k-$ out - of $-n$ systems has the following properties: $R(k) \geq R(k+1), k=0,1, \cdots, n$.

Proof: formula (1) can be written in recursive form $R(k)=R(k+1)+\sum_{x \in \Omega(k, n)} \psi(x)$. Since $\sum_{x \in \Omega(k, n)} \psi(x) \geq 0$, then $R(k) \geq R(k+1)$ is proved.

So far, it can be seen that the reliability of the obstacle avoidance system can be expressed as the mean function of the reliability of each sub member. Therefore, the reliability of the whole system can be reflected by changing the reliability of the sub behaviors in the system.

\section{System debugging and analysis}

\subsection{On-board sensor accuracy test}

In order to verify the effectiveness and real-time of snake-like rescue robot obstacle avoidance algorithm based on multi-objective optimization. Using the snakelike rescue robot DK-Snake-II developed by the 
embedded Laboratory of Xi'an University of Science And Technology, the obstacle avoidance navigation test has been carried out. The snake-like rescue robot head equipped with infrared sensors and visual sensors to obtain obstacle distance information within $80 \mathrm{~cm}$ by infrared sensor ${ }^{[11]}$, on the basis of the obstacle avoidance behavior conduct dynamic adjustment.Figure 4 shows the obstacle avoidance process, a set of infrared sensor ranging error statistics. The maximum distance of infrared sensor is $80 \mathrm{~cm}$, the effective range is $25 \sim 45 \mathrm{~cm}$, and the range error is $5.62 \mathrm{~cm}$. The range of small range error can ensure that the rescue robot avoids collisions due to odometer or visual error, and improves the security and robustness of obstacle avoidance.

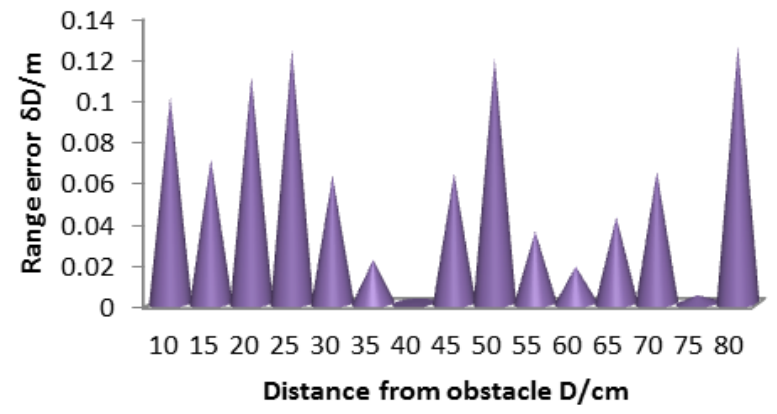

Fig. 4. Infrared ranging error distribution map

\subsection{Obstacle avoidance process analysis based on different decision algorithms}

Snake-like rescue robots use four different decision methods to avoid static obstacles, as shown in figure 5.

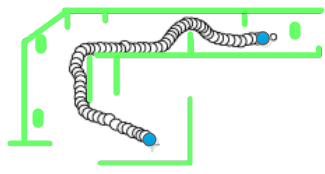

(a) Weighted method

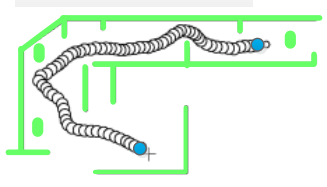

(c) Goal programming method

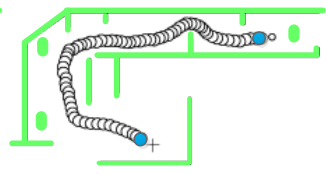

(b) Interval weight target planning method

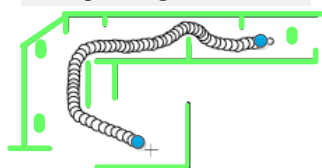

(d) Hierarchical sequence method
Fig. 5. Different decision-making methods obstacle avoidance algorithm to avoid static obstacles

From the figure, the interval weight goal programming method and hierarchical sequence method of obstacle avoidance trajectory is smooth, but the hierarchical method and the weighted method of obstacle avoidance radius is too small, the process of moving the robot velocity and angular velocity are not easy to control, easy to collide, While the interval weight goal programming method has long moving distance, but the trajectory is smooth and the safety factor is high. It is easy for the fast moving robot to adjust the body posture. In view of the four multi-objective decision making methods, multiple experiments are carried out in different environments with different obstacle avoidance complexity and different obstacles placed. Table 1 presents the comprehensive performance index of the multi objective optimization obstacle avoidance method. In this table: $A_{b z}$ stands for obstacle avoidance safety; $V_{b z}$ for obstacle avoidance; robot movement speed; $t$ represents avoidance obstacle response time; $C_{b z}$ for obstacle avoidance success; $\phi$ is trajectory smoothing angle.

Table 1. Comprehensive performance of obstacle avoidance based on multiple object optimization.

\begin{tabular}{lcccccc}
\hline Decision Method & $A_{b z} / \mathrm{cm}$ & $\gamma /\left(^{\circ}\right)$ & $V_{b z} /\left(\mathrm{cm} \cdot \mathrm{s}^{-1}\right)$ & $t / \mathrm{s}$ & $C_{b z} / \%$ & $\phi /\left(^{\circ}\right)$ \\
\hline Weighted method & 38.60 & 33.40 & 21.70 & 0.23 & 77 & 3.1 \\
Hierarchical sequence & 36.50 & 44.30 & 29.30 & 0.24 & 83 & 3.2 \\
Interval weighted goal programming & 41.20 & 37.60 & 24.00 & 0.36 & 86 & 3.3 \\
Goal programming & 43.50 & 47.90 & 28.00 & 0.25 & 82 & 3.7 \\
\hline Average Value & 39.95 & 40.80 & 25.75 & 0.27 & 82 & 3.325 \\
\hline
\end{tabular}

\subsection{Obstacle avoidance test analysis}

Figure 6 shows the process of obstacle avoidance snakelike rescue robot based on multi-objective optimization.

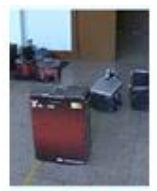

position A

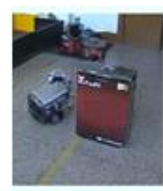

position $\mathbf{B}$

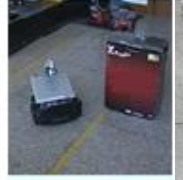

position $\mathrm{C}$

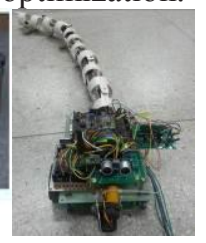

Fig. 6. Rescue robot head obstacle avoidance process During the obstacle avoidance of the snake-like robot head starting from the initial position of $\mathrm{A}$, bypassing the obstacle position $\mathrm{B}$, enter the safe position of $\mathrm{C}$.

Under the control of three sub functions, the robot selects a path which is most suitable for avoiding obstacles and adjusts its pose to the target position.

The position of the three positions relative to the target point, the slip angle and the sampling period are as follows:

Location A indicates $\theta_{0}-\theta_{\text {target }}=0.879 \pi, T=0.045$

Location B indicates $\theta_{0}-\theta_{\text {target }}=0.007 \pi, T=0.052$

Location C indicates $\theta_{0}-\theta_{\text {target }}=0.896 \pi, T=0.039$

\subsection{Reliability analysis of multi-objective optimization output system}

The simulation test of autonomous obstacle avoidance system for snake-like rescue robot with three output behaviors is carried out. Figure 7 gives the mean function curve of system reliability at $\mathrm{k}=5$ and $\mathrm{k}=10$ respectively. Among them, the reliability of each subsystem is equal. That is $P(i)=0.5, i=0,1, \cdots, n$. 

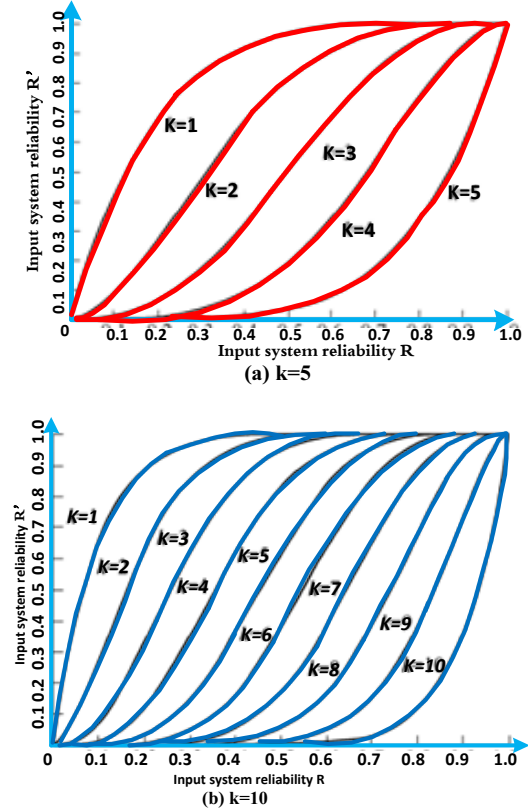

Fig. 7. Multi-behavior output system reliability relation of input and output system

As can be seen in figure 7, after multi-behavior fusion output system reliability can be represented as a function of the input system reliability behavior means, and increases with the increase of behavior reliability mean, or input system with low reliability behavior can be constructed with high reliability of the system output. The larger the $\mathrm{K}$ value is, the lower the reliability of the output system is. At the same mean time, the reliability of the system $k-$ out $-o f-n$ is higher than that of the $k+1-$ out - of $-n$ system when $k=0,1, \cdots, n$.

Therefore, in the obstacle avoidance system based on multi behavior fusion, the proper number of sub behaviors should be determined. It is necessary to ensure the high reliability of the fusion system, and to ensure that multiple sub behaviors cannot bring heavy burden to the system.

\section{Conclusion}

In this paper, the autonomous navigation system for mobile robot due to too much time to seek the optimal path or the optimal solution of deadlock or shock phenomenon, put forward the snake-like rescue robot behavior of a dynamic weighted fusion algorithm of obstacle avoidance.

The algorithm uses the multi-objective optimization method to obtain the most efficient solution of the rescue robot, and divides the mobile robot's obstacle avoidance navigation process into three subsystems. By dynamically changing the weights and priorities of the sub behavior function, the most satisfactory path or the most effective path at the current time is obtained in real time.Experimental results show that the algorithm can effectively improve the security and smoothness of obstacle avoidance navigation, and improve the reliability of the system, while ensuring the robustness of the obstacle avoidance process.

\section{References}

1. SIMMONS R. The curvature velocity method for local obstacle avoidance[C] Proceedings of the IEEE International Conference on Robotics and Automation,Minnesota,Minneapolis:IEEE CNF, 1996:3375-3382.

2. SHI Dongqing,COLLINS E G,DUNLAP D. Robot navigation in cluttered 3-D environments using preference-based fuzzy behaviors[J]. IEEE Transactions on Systems,Man., and Cybernetics, Part B,2008,37(6) :1486-1499.

3. KIRBY R, SIMMONS R, FORLIZZI J. Companion: A constraint optimizing method for personacceptable navigation[C]ROMAN2009, Toyama,Japan:IEEE,20 09:607-612.

4. SEDER M,PETROVIC I. Dynamic window based approach to mobile robot motion control in the presence of moving obstacles[C] Proceedings of the 1996 IEEE International Conference on Robotics and Automation,Roma,Italy:IEEE CNF,2007:19861992.

5. SCHRÖTER C, HÖCHEMER M, ROSS H M. A particle filter for the dynamic window approach to mobile robot control[C] Proceedings 52nd Int. Scientific Colloquium (IWK), Ilmenau,Germany:Universitätsverlag,2007:425-430.

6. NUNKAEW W,PHRUKSAPHANRAT B. A fuzzy multiple objective decision making model for solving a multi-depot distribution problem[C] Proceedings of the International Multi Conference of Engineers and Computer Scientists,Hong Kong,China:IMECS,2010:17-19.

7. SADJADI S J,HABIBIAN M,KHALEDI V.A multi-objective decision making approach for solving quadratic multiple response surface problems[J]. Int. J. Contemp. Math. Sciences, 2008,32(3) :1595-1606.

8. PIRJANIAN P. Reliable reaction [mobile robot navigation][C].IEEE/SICE/RSJ International Conference on Multisensor Fusion and Integration for Intelligent Systems, 1996:158-165.

9. Fu Chao, Zhang Lefeng. Research on autonomous obstacle avoidance for indoor mobile robots [J]. industrial control computer, 2015,28 (07): 44-46+49.

10. Yongyue, Li Shuqin. Research on multi robotic fish formation strategy planning based on [J].Computer simulation, 2013, 30 (11): 369-373.

11. Li Hongyan, Hou Yuanbin. Design of actuator and control system of snake robot based on LPC2132 [J].Journal of Xi'an University of Science And Technology, 2016,36 (02): 265-270.. 